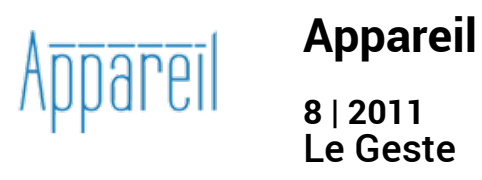

\title{
Les génériques de Saul Bass
}

Joachim Daniel Dupuis

\section{(2) OpenEdition}

\section{Journals}

Édition électronique

URL : http://journals.openedition.org/appareil/1279

DOI : 10.4000/appareil.1279

ISSN : 2101-0714

Éditeur

MSH Paris Nord

\section{Référence électronique}

Joachim Daniel Dupuis, "Les génériques de Saul Bass », Appareil [En ligne], 8 | 2011, mis en ligne le 26 octobre 2011, consulté le 30 juillet 2020. URL : http://journals.openedition.org/appareil/1279; DOI : https://doi.org/10.4000/appareil.1279

Ce document a été généré automatiquement le 30 juillet 2020.

\section{(c) (i) ()}

Appareil est mis à disposition selon les termes de la Licence Creative Commons Attribution - Pas d'Utilisation Commerciale - Pas de Modification 4.0 International. 


\title{
Les génériques de Saul Bass
}

\author{
Joachim Daniel Dupuis
}

1 En 1977, Gérard Blanchard présente à l'école des beaux-arts de Besançon un article remarqué sur Saul Bass ${ }^{1}$, suite à la venue en France de celui-ci, à l'invitation d'un groupe de graphistes français et du soutien de la revue Communications et langages. Il y propose une lecture des génériques et courts-métrages du «designer graphic » basée sur l'idée de logotype, terme qui désigne le discours de la marque propre à un objet design et qui met en évidence la dimension typographique, ou "sémio-typographique » de celui-ci, mais qui suppose aussi l'idée de figure. Saul Bass apparaît ainsi comme le maître d'œuvre d'une normalisation de l'image. On peut toutefois se demander si ce n'est pas Blanchard lui-même qui occulte toute la nouveauté du travail des «titres » de Saul Bass en confondant l'activité créatrice dont il fait preuve avec les nécessités de servir les intérêts d'une marque ou d'une firme. Certes Saul Bass a bien «logotypé ${ }^{2}$ " pour un grand nombre de firmes, mais au-delà de cette activité très contrainte, il crée avec ses génériques, images mobiles, et non figures, quelque chose qui échappe à tout discours, et dont les effets font émouvoir et rendre capable d'expérimenter les images au-delà de toute assimilation à un langage établi ou à un discours. Il y a chez Bass un véritable chef d'orchestre, chaque générique initiant sa marque de fabrique et mettant en avant des gestes de mutilation. Oui, si Bass annonce un ordre, ce n'est pas celui de la normalisation, des appareils technico-industriels et financiers qui s'étaient déjà initiés dans le design au début du $\mathrm{xx}^{\mathrm{e}}$ siècle, mais quelque chose de l'ordre du vampire ${ }^{3}$.

\section{Le logotype}

2 L'intérêt de l'analyse de Blanchard réside dans sa capacité à donner une cohérence à l'univers de Saul Bass, à travers une hypothèse de lecture: le logotype, et en s'appuyant, pour la valider, sur les propos de Bass lui-même (oraux comme écrits) ${ }^{4}$. Cette hypothèse, Blanchard, ne l'exprime pas pour elle-même, il la tient pour connue. Il avait tenu à Lausanne, la même année, un exposé remarqué et remarquable sur le logotype (« de la marque : le logotype ») et écrit une étude sur le collage (« Eisenstein et 
les hiéroglyphes de l'inconscient ») qui ont dessiné les grands principes du logotype. Dans le premier article, le logotype est "l'image de marque » d'une firme et a quatre caractéristiques : un cadre (ou encerclement), le nœud (lettres à boucles ou ornées), l'entrelacement des lettres, la surimpression (figuratif /linguistique).

3 Ces quatre critères, on peut les retrouver dans son analyse des génériques de Bass, ce qui nous permettra de mieux les définir.

\section{1. Premier critère}

4 Le premier critère semble, selon Blanchard, peu respecté dans le monde des génériques, sauf chez Saul Bass, qui a travaillé "à "logotyper" le titre d'un film en fonction de son utilisation dans le générique, dans le matériel publicitaire (affiche, annonce, etc.), sur les pochettes de disque et autres ${ }^{5}$ ». "Idée nouvelle, continue Blanchard, imposée par la prolifération du marché ", « qui consiste à homogénéiser les produits d'une même origine et même à systématiser l'emploi d'éléments communs fixes concernant les diverses exploitations d'un titre. Cette normalisation est ordinairement assurée par le directeur artistique» (Blanchard, 1977). Il y a donc comme une unité graphique, un cadre que Bass semble reproduire et ainsi faire correspondre à une marque de fabrique. Si l'on peut parler de normalisation, c'est au sens plutôt d'une cohérence artistique : il ne suit aucune politique publicitaire. Il ne faut pas confondre un logo et un générique. On peut voir la cohérence de l'artiste qui travaille à rendre compte de son travail sur un « objet » de différentes manières. S'il y a à la limite des encerclements, ils ne sont jamais les mêmes. On ne peut pas dire, mais il faudra le montrer, qu'une pochette de disque d'un film ait le même sens que le générique, dont il prend pourtant un certain graphisme ou des images réelles.

\section{2. Deuxième critère}

5 Le second critère se rapporte davantage à l'ornementation, à la calligraphie de la lettre. Le choix de Bass en général pour une typographie simple, le sans serif, est lié à une influence du Bauhaus. Recherche de la simplicité, découpage des lettres, cette typographie a l'avantage peut-être de permettre à l'émotion de passer plus facilement.

\section{3. Troisième critère}

6 Le troisième critère : l'entrelacement. Dans un générique, il y a deux éléments qui sont à distinguer, les logotypes qui se rapportent plutôt aux lettres, et les pictogrammes qui se rapportent à des images. Pour Blanchard, la syntagmatique de Metz peut illustrer ce niveau. En effet on peut déterminer une échelle : un syntagme long, comme un titre, et à l'autre bout un syntagme ultracourt, qui sera la condensation d'un discours dans une seule lettre (le sigle). Dans son article sur le logotype, Blanchard insiste sur le sigle, mais tous les titres de Bass sont des syntagmes longs, et ne renvoient d'ailleurs jamais à une marque. Même si comme il y est obligé certains de ses génériques seront précédés des logos, des «marques» des grandes firmes, mais ils ne sont en rien liés au générique : ils en prendront même la tonalité (qu'on pense au générique de North by Northwest qui colore en vert le grand logo de la firme). 
7 Blanchard cherche à montrer que c'est l'association image/lettre du générique qui va fonctionner comme logotype. Et pour cela, il convoque un texte de 1961 de Bass. C'est l'entrée sur la scène du symbole. Pour illustrer son propos, Bass évoque dans son article Eisenstein. Celui-ci compare les hiéroglyphes japonais d'il y a 2600 ans avec le travail du montage au cinéma. "L'image de l'eau adjointe à l'image de l'œil signifie pleurer. L'image d'une oreille près du dessin d'une porte signifie écouter ». Blanchard considère que « cette question que pose Eisenstein sur le principe du cinéma et la culture japonaise, avec une digression sur le plan et le montage, Saul Bass la reprend à son compte ». Et que « c'est à partir d'elle que l'on pourrait analyser les différents symboles qu'il nous propose conjointement au titre des films qui les explicitent ou qu'ils explicitent. Par exemple :

- La rose + la flamme = Carmen

- le bras brisé = l'homme au bras d'or

Là commencerait, dit encore Blanchard, une analyse du fonctionnement de ces différents éléments assemblés et de leur rapport au titre, au logotype, au jeu de mots qui sous tend toute une chaîne d'associations simples permettant de les interpréter (Blanchard, 1977).

8 Il faut constater que la citation de Blanchard coupe justement la chose la plus importante: d'une part, il n'est pas fait mention que le titre est toujours plus que la somme des éléments graphiques, autrement dit que ce n'est pas un simple assemblage, une opération additive ${ }^{6}$; d'autre part, il y a assimilation de la notion de symbole à celle de figure (un hiéroglyphe, ou comme disent les égyptologues un idéogramme). L'idée de Bass est au contraire de mettre l'accent sur le mouvement et sur l'idée de rythme qui est différente de la peinture au cinéma: la peinture instruit un séquençage par blocs d'éléments, on peut ici parler d'addition; mais quand le nombre d'éléments à voir est important, il «est nécessaire d'organiser ces différents points une séquence rythmique » (Bass, 1961). Le générique « agit sur le spectateur ». Le « film vit dans son propre espace-temps, sur ses propres données, et le spectateur en subit le mouvement plus qu'il n'en provoque » (Bass, 1961). Le générique n'est donc pas basé sur une association de l'esprit qui viserait à interpréter quelque chose. C'est plutôt l'enchaînement des images, et des lettres dans les images, qui rendent possible un certain temps, un certain mouvement. Le logotype suggère une norme qui imposerait comme un sceau un sens aux figures d'une image en mouvement. Mais le générique s'il est bien animé n'est pas une somme de figures dont le sens serait assigné par un appareil technico-industriel et financier (firme ou industrie du cinéma). Il faut considérer que les images du générique ne sont pas des signes qui renvoient à notre monde et à sa logique politique.

\section{4. Quatrième critère}

9 Le quatrième critère : la surimpression. Celle-ci est abordée dans le texte de Blanchard sur Bass notamment avec un très beau générique: Walk on the wild side (tellement bien fait que les spectateurs allaient le voir sans regarder la suite : le film). Le générique montre la promenade d'un chat noir que nous suivons passant par un tuyau d'écoulement, avançant dans les arrière-cours, jusqu'à son combat avec un autre chat blanc. Les effets de surimpression sont rendus par des glissements d'images, qui semblent insaisissables, comme dans un rêve. Il y a une persistance de l'image qui reste 
présente un certain temps sur l'écran, que Blanchard assimile à l'idée de "tierce image » de Christian Metz. "Seule, en effet, une analyse de la dynamique des images peut rendre compte de ce phénomène de surimpression et des images ainsi formées par leur superposition, et dont le sens n'est pas égal à la "somme" mais à un "produit" complètement différent " (Blanchard, 1977). Nous retrouvons ici l'idée que le sens du symbole n'est pas une addition simple (ce qui conteste le critère précédent), mais surtout on se demande comment on peut encore être dans du logotype si nous touchons à autre chose que du réel. Pour Blanchard, la condensation et les déplacements des images rendent compte d'un effet de sens, comme le discours d'un inconscient. Son explication est laconique: "la conjonction du film court, des motivations allant jusqu'aux images de l'inconscient, à la limite des images subliminales (imperceptibles) et de la beauté des objets, est à l'heure actuelle commanditée par des firmes commerciales » (Blanchard, 1977).

Les analyses de Blanchard, dans la mouvance de Christian Metz ${ }^{7}$, dessinent une pensée du figural, notamment rendu possible par le dispositif du logotype et un certain goût pour une économie psychique proche des déplacements et des condensations de Freud. Il distingue au demeurant trois phases du logotype dans les génériques de Bass: une phase d'abord esthétique (faites de symboles vignettes, qui anime l'espace en lieux du texte), une deuxième période, intégrant non plus un graphisme, mais des prises de vues réelles, et une troisième qui est celle de la surimpression. Blanchard dessine ainsi les métamorphoses du générique de Bass qui s'adapterait à des nouvelles contraintes du marché, notamment en rendant compte de la part de rêve - d'où l'art de créer des effets de persistance rétinienne sur l'écran. Mais est-ce que cette explication plus sociologique qu'artistique est à même de nous satisfaire?

\section{Contre Blanchard}

Il est tout à fait frappant de voir que Blanchard met de côté dans son analyse la seconde partie et un morceau essentiel de la troisième partie de l'article de Bass de 1961, mais aussi ne convoque pas du tout les entretiens de Bass que l'on peut lire la même année où Blanchard présente sa conférence de $1977^{\circ}$. Or la lecture de ces textes suggère une autre hypothèse de lecture des génériques de Bass. Non pas qu'il n'y ait pas de logotypage, mais l'importance que lui accorde Blanchard semble laisser penser qu'un générique est un produit industriel, un produit d'une firme, d'une commande. Or c'est là peut-être un paradoxe, mais si les firmes du cinéma ont donné les moyens de produire des génériques et des films à des réalisateurs et aux designers, elles n'expliquent en rien la composition des génériques eux-mêmes. Il ne faut pas considérer que la marque imprime à l'art ses directives, quand c'est précisément l'art qui va renflouer l'intérêt pour le cinéma, qui dans les années où débute Bass au cinéma, subit un essoufflement ${ }^{9}$. Hollywood, dans les années 1950, donne les conditions d'une créativité cinématographique qu'elle ne cherche pas à étouffer dans une normalisation, même si celle-ci finira par gagner le générique à un moment où Bass ne fera presque plus de génériques mais des courts-métrages (fin années 1960). Bass fait des génériques non pas des types tout faits (cartons), mais des œuvres d'art, des créations à part entière, il insuffle aux firmes la nécessité d'intégrer en quelque sorte un aspect essentiel: l'émotion. Nous proposons donc de lire les affects produits au cinéma comme des gestes de mutilation, une analyse graph'ique, mettant en œuvre toute une imagin-action. 

quelle en 1965, dans l'ouvrage Signe, image, symbole, sous la direction du grand maitre de Bass, György Kepes, où il décrit son intérêt pour le générique, propose une définition du média, et témoigne des similitudes entre le cinéma et la peinture. La seconde partie développe par l'exemple la dernière idée de la première partie - que la peinture a quelque chose qui l'apparente au cinéma par le mouvement. C'est aussi la première référence faite à Eisenstein (tiré de son livre Film Form). Bass retient surtout la référence aux carnets de Léonard de Vinci par Eisenstein, car elle indique que le montage est une préoccupation ancienne, qu'on retrouve dans la peinture de la Renaissance. Il y a ainsi nécessité de penser l'image picturale en séquences narratives, quand l'image décrite est trop complexe. Mais la troisième partie - que Blanchard reprend en partie dans son texte sur Bass de 1977 - illustre l'idée du symbole selon Eisenstein, mais surtout va plus loin que ce qu'il en dit. Il s'agit pour Bass de mettre en évidence que le symbole cinématographique n'a pas besoin du texte pour être compris et que le spectateur doit «expérimenter le film plus que l'entendre ou le voir ». Et cela passe par «le potentiel émotif du procédé». Il est clair que Blanchard ne pouvait intégrer cette idée dans son analyse car elle l'aurait simplement anéantie. En effet, si c'est l'émotion qui domine dans l'image, un dispositif visant à encercler, lier, entrelacer, sur-imprimer ne peut pas produire que des signes transparents, rationalisables: chercher à interpréter un générique, selon ses signes normalisés, plutôt qu'à le vivre, ou l'expérimenter, relève donc d'un processus de lissage de l'émotion, donc de sa disparition: on ne programme pas des affects. Pour Bass, la compréhension ne passe plus par la communication, par le discours, dût-il être inféodé à un dispositif logotypique. Elle va même plutôt le défaire, le mutiler, travailler à sa mise en échec. Blanchard a précisément voulu normaliser à son tour le travail de Bass, mais ce faisant, il en a occulté l'essentiel.

Plusieurs indices permettaient pourtant d'éviter pareille erreur.

\subsection{Premier indice}

Le premier indice tient à la définition même du générique donné par Bass.

La première partie de l'article de 1961 offre une définition du générique tout à fait nouvelle. Tout d'abord l'insistance à sortir le spectateur de son attente, de sa causerie, ou de son activité de manger (Pop-corn). Il fallait qu'« il se passe réellement quelque chose sur l'écran ». Saul Bass pose ainsi la définition du générique : «je pensai qu'il était possible de projeter un avant-goût symbolique de ce qui allait suivre ». Cette définition sera plus clairement rapportée à l'idée d'affect, en 1977, dans son fameux entretien visuel : «Bass on titles ». Il y parle de « créer un climat pour l'histoire sur le point de se déployer ». Le générique n'est donc pas le début narratif du film, mais il est une sorte de "temps». Le générique offre donc une coupure, ou plutôt une morsure. L'intérêt pour le film ne vient pas de l'histoire, mais de ce qui se projette avant. Si le film est l'événement, le générique en est l'écho. Le générique se présente d'emblée lié à un certain futur, que nous allons voir avec le film, mais il n'en est pas le miroir. L'affect est donc orienté, il se donne lui-même selon une certaine disposition de l'image, comme à travers l'activation d'une membrane, au sens où l'entend Simondon ${ }^{10}$. L'image est en effet orientée selon un certain temps, une direction et en même temps montre quelque chose. 
Mais alors pourquoi parler du générique comme d'une partie du film lui-même si celuici contient les éléments mêmes du grand organisme (article de 1965) : «alors que le titre du film constitue une portion assez petite pour le grand organisme qu'est le film, je découvris qu'il contient en miniature beaucoup de procédés et de problèmes qui sont caractéristiques du film même ». Il faut donc concevoir que l'image du film n'est pas différente de l'image du générique dans ses aspects visuels, auditifs, que dans sa forme, le générique est bien une partie d'un film, mais aussi que ce générique pourrait aussi passer dans le film, intervenir à un moment donné. Ce qui diffère, c'est donc non pas l'image, à proprement parler, mais le travail opéré sur elle, et sur ce qu'elle représente, son contenu. Pour le dire plus clairement, ce qui distingue l'image du générique, c'est que c'est un symbole.

\section{2. Deuxième indice}

Le second indice, c'est l'idée de symbole. S'il y a symbole, c'est qu'il y a signe dironsnous. Mais signe de quoi ? Si le mot symbole est utilisé, c'est en référence à la tradition du Bauhaus, dont Saul Bass est issu : mais nous venons de voir que le générique ne peut pas renvoyer au film, puisqu'il est une partie du film. Il n'est pas non plus index sui, puisque quelque chose du film passe en lui. Si donc le symbole a perdu sa dimension de signe conventionnel, ne peut incarner le sens archaïque de deux moitiés que l'on peut reconnaître ensemble (sens étymologique), il faut peut-être penser que le symbole est plus à prendre dans son sens actif, de ce qui est « jeté-avec ».

18 Un entretien téléphonique de $1993^{11}$ peut nous aider à répondre. "À mon avis, la première image d'un film et son générique présentaient la possibilité de créer une ambiance [...] un autre point important, c'est l'élément graphique du film qui le présente ». On pourrait penser que le symbole et l'affect sont deux choses différentes, mais il n'y a pas d'affect sans symbole. C'est précisément la mise en mouvement du symbole qui va créer le premier générique : Bass insiste à chaque interview sur la mise en mouvement de ce symbole de "Carmen ». L'affect est donc indéfectiblement lié au mouvement même du symbole, à la mobilité de l'image. À un geste. Le symbole est en fait à la fois quelque chose qui est en mouvement, mais aussi quelque chose qui configure. Ce qui se donne dans l'image n'est pas une figure en mouvement ni un signe, mais l'opération d'un geste.

19 "Le générique est (donc) un avant-goût symbolique de ce qui va venir » (Bass, 1961). L'oxymore entre symbole et affect traduit bien leur lien, ou leur consistance réciproque, qui ne se réduit pas non plus à un rapport d'identité, de copie.

\section{3. Troisième indice}

20 Le troisième indice concerne la notion d'événement. Si le générique présente quelque chose comme la mise en œuvre d'un geste qui se configure en déployant des traits graphiques (pour les premiers génériques), mais aussi des traits de déformation, ce geste doit pouvoir être attrapé et on doit pouvoir le retrouver dans n'importe quel générique. Mais ce geste doit être l'écho d'un événement, de quelque chose qui n'est pas là et qui va s'actualiser dans la suite du générique. C'est comme si le générique en était le «retentissement ", la suspension virtuelle, comme l'élément qui vient nous arracher à notre mondanité et nous envelopper d'intensités qui vont nous rendre apte 
à nous confronter à l'événement lui-même. Le générique est donc ce qui porte le choc de l'image, ce qui sera nécessairement violent pour nous porter à arrêter de parler, de boire, de penser à tout et à rien. Le générique a donc un certain potentiel et ce «potentiel émotif du procédé », comme dit Bass, de l'appareil-générique, c'est celui-là même que le film va actualiser à sa façon dans un récit, comme si le générique contenait le film en un point, le condensait. C'est comme si le film justement était aussi ce qui allait «neutraliser » l'effet produit, nous ramener à une sorte de discours, de narrativité, dont le générique justement nous prive. On comprend pourquoi toute l'analyse figurale liée au discours chez Metz et reprise par Blanchard à travers la notion de logotype, à son époque, encore mal définie, est insatisfaisante.

Il nous semble maintenant nécessaire de montrer par plusieurs exemples l'étendue de cette gestualité du générique pensé par Bass, mais exprimée dans un vocabulaire idéologique de son temps, de sa formation. En montrant plus précisément comment le geste du générique est d'abord un geste de mutilation, qu'il va intensifier encore plus à travers le concept de time before, dont Vertigo est certainement le plus exemplaire, nous montrerons l'originalité de la conception symbolique de Bass qui est chez lui l'équivalent d'un diagrammatisme avant l'heure.

Mais d'abord il convient de montrer que les génériques de Bass sont classés et que de cette classification va dépendre des types de gestes.

\section{Classification des génériques}

\section{1. Classification de 1977 en 5 phases}

En 1977, dans Bass on titles, Saul Bass se met en scène dans un entretien où il parle de son travail de designer pour le cinéma. À partir de quelques génériques choisis parmi une bonne quarantaine faits à l'époque, il distingue cinq phases de son étude. La distinction est à la fois technique et intellectuelle. La première phase, incarnée par le célèbre Homme au bras d'or, est la phase graphique ; la seconde phase, que met en scène L'Opération diabolique, est la phase réelle (où le générique est fait à partir de prises de vues réelles) ; la troisième phase consistait à coller au plus près du film : le générique, par sa longueur, pouvait ainsi retracer à sa façon le film, comme dans West Side Story. Mais le plus important était les deux dernières phases : car il ne s'agit plus seulement de symboliser le film ou de faire tomber le spectateur dans un certain climat, le générique peut apporter plus, jouer sur le sens du récit ou rendre le côté infraordinaire d'une chose (pour parler comme Perec). La quatrième phase insiste donc plutôt sur le côté narratif: dans Grand prix, Saul Bass montre le départ d'une course pour elle-même, et dans The Big Country, il s'agit de marquer la solitude qui va entourer les personnages de l'histoire, à travers l'arrivée d'une diligence dans une ville entourée de plaines désertes. La cinquième phase, tournée vers le symbole lui-même, est considérée comme la perspective la plus stimulante pour Bass : L'horloge de À neuf heures à Rama, "traite des neuf heures qui précédent l'assassinat de Mahatma Gandhi. En prenant une horloge, objet ordinaire, et en lui faisant subir un examen acharné, je cherchais à intensifier la conscience en chacun de chaque moment ». 


\section{2. Classification de 1993 en 2 phases}

En 1993, Saul Bass distinguera plus facilement deux phases dans son travail qui pourraient correspondre à la coupure que nous indiquions entre les trois premières périodes et le time before: d'abord, selon cette nouvelle classification, il s'agit de symboliser le film, l'idée du film, sans toutefois que ce symbole soit une expression abstraite, mais plutôt une mise en scène d'une série de traits, d'éléments, dont le sens est irréductible à un signe ou à un ensemble de signes, produisant ainsi un affect. L'affect d'un geste. Puis, une phase, où la neutralité n'est plus de mise, où il s'agit de saisir le time before, de modifier le sens du film, ou encore de mettre en valeur le symbole même du générique.

\section{Les gestes de mutilation}

Pour donner une idée plus claire de la différence entre ces deux phases du traitement du symbole, qui est geste, et qui est le geste d'un certain affect, prenons d'abord trois exemples qui illustreront l'idée que c'est bien un seul geste qui travaille, au-delà des différences techniques, la phase « neutre » du travail de Bass (Bass, 1993).

\section{1. Générique graphique}

Premier exemple: Anatomy of murder. C'est un générique graphique de la première période (1977). L'erreur serait de regarder les images d'un générique à l'arrêt. Sans mobilité, nous ne pourrions pas voir ce qui se joue en quelques images, dans ce dispositif des images en action, dans cette imagin-action. La membrane cinématographique est imagin-action. Que voyons-nous pendant tout le temps de ce générique ? D'abord la constitution progressive d'un corps-silhouette (semblable à un mannequin démantibulé), puis sa dé-constitution. Mais ce qui change entre l'une et l'autre phase du générique, c'est que la fragmentation de l'image n'est pas opérée de la même façon. La constitution prend tout le corps-silhouette en son entier, alors que sa dé-constitution, prend en compte seulement certaines parties du corps, en gros-plan. Deleuze a bien vu que l'affect est visage et que le visage au cinéma est gros-plan. Bass ne le dit pas mais nous le montre. L'efficacité de ce geste de décomposition est telle que ce qu'il montre n'est pas une victime, comme le suggérait seulement la première phase, mais une victime-assassin, comme le suggère les derniers plans sur les mains qui semblent vouloir assassiner le spectateur. Le geste de mutilation est donc comme un geste bi-face, car il ne dessine pas une figure, mais rend compte d'un mouvement qui configure selon un point de renversement (un zéro) un titre dont le sens ne se réduit pas à l'idée de l'autopsie d'une victime (du regard neutre d'un spectateur, comme le regard d'un médecin sur sa victime) mais va plus loin, en suggérant que ce que nous voyons pourrait nous atteindre, que la victime pourrait faire de nous des victimes. L'affect nous rend capable de suivre le film en nous enveloppant et en retournant le sens de l'image en son contraire. Dans le film, il y a l'idée d'une enquête - dans un procès - à propos d'une victime qui est mise à la question. Le film n'explique pas le générique et inversement. Mais il nous y plonge d'une certaine façon. 


\section{2. Générique « réel »}

Deuxième exemple : L'Opération diabolique (Seconds). C'est un générique « réel », de la seconde période (1977). C'est un générique troublant, sans doute la musique du de Jerry Goldsmith y est pour beaucoup. Que voyons-nous? Un visage qui n'a de cesse de se déformer (œil, oreille, bouche), ou d'être montré sous des angles improbables. Ces zones de déformation nous rendent incapables de dessiner les contours d'une figure, on est là face à quelque chose de proche des expériences de Francis Bacon. La surface de l'image est une membrane, elle ne laisse transparaitre que des trous, des lignes, des traits : ce n'est pas le tissu d'une peau où des signes peuvent s'imprimer, une figure se dessiner, c'est une couche disparate. À la fin dans le générique nous voyons un visage cagoulé, un sans-visage. On atteint donc le sommet de la défiguration. L'image finale renverse le début. On a quelque chose comme une naissance qui semble s'être accomplie ; le sans-visage signifiant un autre visage. L'opération de mutilation est cette fois directement sur un visage et elle vise à symboliser, à rendre le geste d'une mutilation et son annihilation. Les deux bords du film tiennent non par opposition simple, mais par tout un processus gestuel qui les rend possibles et neutralise le sens que l'on est d'ailleurs incapable de formuler : ce qui est précisément l'affect. L'affect est comme une sorte de lieu du visage qui se virtualise en lui, c'est une membrane qui laisse passer l'événement, des singularités. Une expressivité.

\section{3. Long générique}

Troisième exemple : West Side Story, c'est un " long » générique et « homographique ». Il y a en fait deux génériques de ce film. Le générique de fin est décrit comme un "sas ", ou une "chambre de décompression" par Saul Bass. Le film produit-il encore une mutilation? Oui, cette fois elle est à deux niveaux. Elle passe au niveau supérieur entre le film et le générique par ses deux bords (début /fin): ce que l'on voit dans le générique, c'est un environnement visuel identique à la rue, à la vie urbaine que le film dessine, il faut donc que le spectateur s'habitue progressivement à voir les graffitis et qu'il se glisse dans ce monde urbain, il doit se glisser dans une rue, un quartier, et il faut aussi que le spectateur après le meurtre violent des personnages puisse «reprendre ses esprits »: d'où la volonté d'arpenter encore des rues où le temps, la longueur des génériques fera son effet. Mais au niveau inférieur, dans chacun des génériques, on retrouve le découpage du mur qui présente les facettes des noms des protagonistes, et créditent les participants du film. C'est la fragmentation d'un "visage ", sa membra-nisation.

\section{Time before}

Maintenant que nous avons pu montrer qu'un seul et même geste travaillait bien les génériques de la première phase (toujours selon la classification de 1993), il convient de bien faire voir ce qui change avec la seconde phase (le time before). L'exemple de Vertigo nous semble le plus difficile mais aussi le plus caractéristique. 


\section{1. Question d'équilibre (entre générique et film)}

On lit d'ordinaire les génériques par rapport au film. C'est-à-dire que nous trahissons précisément sans nous en rendre compte le travail même du générique sur nous : nous ne regardons pas un générique (de début), en fait nous y revenons toujours après-coup, après le film, ayant oublié la découverte des premières images. Dans le cas du film d'Hitchcock, Vertigo, on peut dire qu'il est peut-être le générique qui a donné lieu au plus grand nombre d'interprétations.

Vertigo est peut-être l'incarnation la plus remarquable de ce concept du time before que Bass a déployé de plusieurs façons. Pris dans son second aspect (cinquième période de 1977), le time before déploie le symbole pour lui-même, en nous le faisant voir autrement. De quel symbole s'agit-il ? Du symbole du « vertige ${ }^{12}$ ».

Le vertige dans Vertigo est souvent pris comme la désorientation spatiale de Scottie. On voit dans la jeune femme montrée au tout début du générique, la figure de Madeleine ou de Judy, bref, on se met à interpréter le générique par rapport au film. Si on se réfère à l'idée du time before, on doit rompre avec tout rapprochement avec le film. Mieux, si c'est bien un générique de type time before, alors ou il va aller plus loin que le film en nous donnant une sorte de clef du film (ce qui n'est pas le cas ici), ou le générique va se lover sur lui-même, et nous ouvrir autrement au symbole qu'il porte et qui est dans le film, «incarné » par le motif des spirales. Pour notre part, il est un générique où le symbole "exprime " plus que dans le film. Il est membraneux, alors que le vertige dans le film est lisse et peut se décliner selon les personnages (Scottie en étant l'objet, mais les personnages ou les lieux le motif).

Réduire le symbole du vertige du générique au vertige du film, c'est retomber dans le sens de la chute, la chute de Scottie (réelle, l'accident du début, ou figurée, amour pour une madeleine virtuelle, dont on sait qu'elle est jouée par Judy). C'est donc reterritorialiser le générique sur le film. C'est d'ailleurs pourquoi Bass articule l'idée du vertige avec une femme, pour éviter de « chuter » dans une interprétation.

4 Le symbole du vertige, selon Bass, ne doit pas avoir un sens mais faire sens, avec toute cette dimension affective que cette expression verbale contient. Bass aura donc voulu saisir la profondeur du vertige qui n'est pas que spatiale, qui est affective, au sens plein où il tient du visage et de ce qu'il exprime au plus profond de nous. Il s'agit donc de penser le vertige tel qu'il se donne dans sa plus grande amplitude, qui n'est pas une désorientation seulement de l'équilibre physique (de l'oreille interne, ou que sais-je ?), mais quelque chose qui nous arrache à toute terre, qui nous déleste de toute masse ${ }^{13}$.

\section{2. Les gestes de Vertigo}

À vrai dire, le générique va nécessiter deux gestes, plutôt qu'un seul. Le générique déploie deux gestes, une membrane complexe : toute la force du générique réside en ce passage d'un geste à un autre. Il y a tout d'abord le geste de mutilation (qui d'ailleurs aboutit exactement à une mutilation de l'œil) et le geste de régression (un approfondissement de l'affect, qui est rendu par des opérations graphiques de désorientation).

Tout d'abord, le travail sur une prise de vue réelle est celui d'une caméra. La caméra encercle et tourne autour - dans un mouvement lui-même mutilant, puisqu'elle semble 
découper le visage - l'espace d'un visage, celui d'une jeune femme (que nous ne pouvons assimiler à personne puisque nous n'avons pas vu le film et qui n'est d'ailleurs pas l'actrice principale du film). À un moment, la caméra encercle les yeux qui semblent osciller de droite à gauche, suggérant clairement que la caméra est en fait une sorte de miroir où le visage de la jeune femme se regarde : c'est le niveau de symétrie (car il tient ensemble le visage réel et son image virtuelle: elles sont indiscernables pour nous comme pour elle). Du moins cette indiscernabilité n'est-elle pas celle d'une réplique de l'image, d'un effet-miroir. Ce moment est en effet un moment de frayeur. Ne dit-on pas que les yeux sont les portes de l'âme ? La désorientation commence, dans le sens, où la jeune femme se voit se regardant, elle n'est plus en phase avec elle-même, mais comme en face d'elle-même sans se reconnaitre complètement, ou de quelque chose d'elle-même qui lui échappe (commence la dissymétrie). Cet écart est précisément ce qui explique la terreur, la frayeur, le frisson qui saisit la jeune femme. Elle commence à se voir comme une autre. Le vertige est donc tout autant spatial (visuel) que temporel, car elle est n'est plus attachée à l'ordre du monde et des heures, au fil du temps terrestre. Elle se sent partir. La mutilation du geste de la caméra porte donc ici directement un effet au niveau de l'œil lui-même que nous voyons devenir rouge. Le moment est au maximum dans la membrane de l'œil. C'est la marque de l'affect qui touche la jeune femme. Et ce qu'elle voit, nous allons le voir aussi.

Commence alors un second geste, qui semble s'effectuer à travers l'œil, à un niveau de réalité qui est la conscience, ou plutôt la mémoire de la jeune fille. Le passage aux images de synthèse graphiques explicite clairement le symbole de la spirale, proposé d'abord on l'a vu, comme un mouvement réel de désorientation sur le visage. Maintenant le symbole est explicite, mais il est sans masse. Il n'y a plus de point d'ancrage de la caméra. Le spectateur est attrapé par un espace topologico-affectif : d'où la modulation des couleurs qui accompagnent les oscillations. C'est un véritable espace riemannien (d'ailleurs il correspond ondulatoirement à des ondes de Lissajous). On peut discerner dans ce long moment spiralaire, un nœud de Moebius, une bouteille de Klein. Ce geste qui s'opère est un geste de régression, car il s'agit précisément de faire vibrer le point de l'œil, de voir en quoi il n'est pas un point mais un trou. Le geste n'est pas celui d'une spirale, à dire vrai (ce en quoi le film va actualiser une image spiralaire qui ne rendra pas toute l'intensité du générique) mais plutôt celui d'une vrille, d'un vissage à l'infini, qui par sa complexité atteint des niveaux topologiques intéressants, comme le nœud de Moebius, où il n'y a plus d'intérieur ou d'extérieur. Car précisément nous sommes dans un espace-temps différent, celui de l'affect, tourné vers le temps.

Mouvement rotation/translation qui meut le vertige, le symbole du vertige en espace de subversion de toute transitivité, comme dirait Châtelet. Le geste de régression ne mutile plus, il creuse et dessine une désorientation qui engage le temps lui-même.

Puis nous revenons au visage et la vrillation se défait, comme si nous revenions sur terre, après avoir parcouru tout un espace d'étrangeté. L'effet nous aura donc emportés nous aussi et pas seulement la jeune fille qui vit pourtant sa propre dé-figuration.

Vertigo est donc un générique complexe car le time before n'est pas seulement celui qui précède le film, le narratif (où le vertige aura cette fois une intensité contextualisée, mais aussi se vivra, au long du film, dans son déploiement amoureux ou peureux), mais un temps avant toute mondanité, temps-espace, blocs d'espace-temps qui nous échappent et qui font que nous touchons à un devenir-animal. Non pas que nous devenons autre chose, ou que nous aurions une part animal insoupçonnée qui se déclarerait dans des 
moments exceptionnels, mais nous subissons comme une morsure, la morsure d'une sorte de vampire qu'est le temps, le temps n'étant pas lui-même une figure, mais une sorte de brassage ${ }^{14}$, qu'aucune chronologie serait bien incapable de retrouver. Le générique se constitue selon une dissymétrie qui est comme le circuit du virtuel sur l'actualisé et de l'actualisé sur le virtuel. Comme une sorte de circuit qui est un passage à la limite, un moment de déséquilibre. Nous passons du déchirement de l'œil, orienté vers la perception aux spectralités de la lumière intérieure, de l'âme, de l'esprit, de la mémoire. Ce basculement est rendu possible par un écart (mutilation) et par un certain tourner-autour (vrilles du temps).

41 Ce que Bass nous a apporté c'est donc une certaine façon de toucher à ce time before. Il n'y a qu'à espérer que maintenant les génériques seront expérimentés comme Bass l'a imaginé. Il est en tous cas certain que c'est le manque d'imagin-action devant les expériences de pensée - les expérimentations qui ont lieu dans les génériques - qui nous fait ne plus les regarder et qui est donc à l'origine du déclin de ce média au profit du pré-générique, qui n'est qu'une scène pleine d'action et d'émotion, mais qui ne renvoie à aucune étrangeté.

Cette conception du générique de Saul Bass modifie complètement la manière de voir le film, mais aussi l'idée que l'on peut se faire du film lui-même. À cet égard, Phase 4, le seul long métrage de Bass, produit en 1974, et qui lui a valu une certaine reconnaissance des spécialistes, est d'une certaine façon un film-générique. Le générique contamine, parasite le film sur toute sa longueur. Après le générique, c'est donc le film qui doit se défaire de la profusion de paroles et nous affecter.

Considéré dans son ensemble, le travail de Saul Bass fait du générique beaucoup plus qu'une simple "ouverture» du film; il en fait, au sens deleuzien, une véritable « machine de guerre » capable de nous faire voir autrement le cinéma.

\section{BIBLIOGRAPHIE}

Blanchard Gérard, « Saül Bass : génériques et films », Communication et langages, vol. 40, $\mathrm{n}^{\circ} 40$, 1978, article accessible sur le site Persée : http://www.persee.fr/web/revues/home/prescript/ article/colan_0336-1500_1978_num_40_1_1255.

Bourget Jean-Loup, Hollywood, la norme et la marge, Paris, Armand Colin, 2005.

Fain Gérard, De l'équilibre au déséquilibre, d'où vient le vertige, Paris, Le Pommier, 2010.

Lupton Ellen, Thinking with type, New York, Princeton architectural press, 2010.

Metz Christian, Le signifiant imaginaire, Paris, Christian Bourgeois éditeur, 1984.

Roy Philippe, « Geste cinématographique et cinéma documentaire », Appareil, nº 6, 2010.

Simondon Gilbert, L'individuation à la lumière des notions de forme et d'information, Jérôme Millon, 2005. 


\section{NOTES}

1. Cf. Gérard Blanchard, "Saül Bass : génériques et films », 1977 : article accessible sur le site Persée.

2. Selon Ellen Lupton, logotyper, c'est "uses typography or lettering to depict the name or initials of an organisation in a memorable way [...]. A logotype uses words and letters to create a distinctive visual image [...] A logotype is part of an overall visual brand, which the designer conceives as a 'language' that lives (and changes) in various circumstances", in Thinking with type, New York, Princeton architectural press, 2010, p. 68.

3. La notion de vampire n'est ici ni prise comme figure (Dracula) ni pensée comme métaphore (comme chez Marx qui en fait une métaphore du capital), le vampire renvoie plutôt au temps luimême, comme on le comprendra mieux à la fin de l'article avec l'exemple de Vertigo.

4. L'inconvénient, disons-le maintenant, est que ce sont des propos tronqués : Blanchard se fait traduire les paroles de Saul Bass qui, dans le cadre du festival où il est invité en France, ne seront pas trop développées, et plutôt anecdotiques; et il ne tient compte que d'un texte de 1961, dont il ne retient que certains aspects, comme nous le verrons.

5. Toutes les citations de Blanchard, sauf cas contraire, sont tirées de son article sur Bass de 1977. 6. Saul Bass dit clairement qu'« on peut envisager ce phénomène, non comme une simple somme mais plutôt comme un phénomène par lequel le tout se trouve être plus important que la somme des parties » ("Propos d'un graphiste sur la bande animée », traduction : Raymond Gid, 1961).

7. Cf. Christian Metz, Le signifiant imaginaire, Paris, Christian Bourgeois éditeur, 1984.

8. Pour cette période, il en existe deux en anglais ; un entretien visuel : « Bass on titles » (1977) et un entretien écrit dans « American cinématographer » : the " compleat film-maker » - from titles to features (1977). Le premier est disponible sur le site http://www.pyramidmedia.com/. Tous les extraits suivants sont traduits par moi-même ou par Yann Serizel.

9. Nous pensons aux analyses de Jean-Loup Bourget : « Hollywood, la norme et la marge », Paris, Armand Colin, 2005.

10. Cf. Gilbert Simondon, L'individuation à la lumière des notions de forme et d'information, Jérôme Millon, 2005, p. 225-226. Chez Simondon la membrane (d'un organisme) est pensée selon des critères que l'on retrouve dans le générique : $1^{\circ}$ ) «La membrane, dit-il, est polarisée » (comme le générique, partie d'un « organisme » qu'est le film, est tourné vers le film, vers l'actualisation, ou vers le symbole que le générique contient), mais cette polarisation est au moins autant temporelle que spatiale; $2^{\circ}$ ) «elle est vivante parce qu'elle se polarise toujours » et que «le vivant (y) vit à la limite de lui-même, sur sa limite ». Le geste du générique est comme un parasite qui vit sur la limite de l'organisme du film; c'est aussi bien comme le film qui vivrait à sa limite, comme affect-symbole; $3^{\circ}$ ) «(Cette) structure vitale la plus primitive et la plus profonde est topologique ». D'ailleurs le concept de membrane que nous utilisons est aussi appelé mem-branique, car il se donne dans une fragmentation, comme pluralité d'éléments (on peut entendre l'idée de «membres »- dimension vitale - comme l'idée des « branes »- espaces-temps).

11. Il s'agit à notre connaissance d'un des derniers entretiens importants de Saul Bass (par Frédéric Temps) avant sa mort en 1996. On le trouve sur le site http://www.generiquecinema.net/histoire/saulbass.html

12. Il est intéressant de lire ce qui se dit sur le vertige en général, voir le petit livre bien fait de Gérard Fain, De l'équilibre au déséquilibre, d'où vient le vertige, Paris, Le Pommier, 2010. Il est évident que la perspective thérapeutique de ce livre n'est en fait en rien exprimée dans le générique de Bass, mais il demeure un contraste saisissant.

13. On ne saurait que trop renvoyer à l'article de Philippe Roy, "Geste cinématographique et cinéma documentaire ", revue Appareil, $\mathrm{n}^{\circ} 6,2010$, qui exprime remarquablement, selon une conception gestuelle plus liée au film, il est vrai, la dimension ondulatoire de l'image, que nous appelons membrane (et qui renvoie à une sorte de théorie des cordes, de l'émotion). 
14. Il faut lire le beau livre de Philippe Roy, L'immeuble du mobile où il exprime cette idée du temps qui brasse et qui est lié à du physico-mathématique.

\section{RÉSUMÉS}

Nous proposons ici de faire connaître la conception du générique de Saul Bass, reconnu à juste titre comme l'inventeur du générique moderne dans les années 1950 aux États-Unis et comme l'auteur de 60 génériques dont l'époustouflant Vertigo. Bien que la notion de geste ne soit jamais utilisée par Saul Bass, nous proposons de montrer qu'elle est à la source des opérations de pensée (symbole) et d'affect de chaque générique. La notion de geste permet de rompre tout autant avec la notion de symbole comme figure et comme métaphore du film qu'avec l'idée de logotype souvent employé dans le design. C'est un dispositif, un appareil ou encore une sorte de membrane où le film se fait l'écho. Son sens ne peut donc jamais être que suspendu à un geste qui ne se laisse pas actualiser. Cet article se veut lui-même comme un long générique sur le reste du travail de Bass : les story-boards de Psycho, les courts-métrages et surtout Phase 4, le grand long métrage où culmine son savoir-faire. Tout prend sens par rapport à ces gestes du générique qui viendront dans son travail de cinéaste " contaminer ", " parasiter » la continuité narrative, et lui insuffler une dimension éminemment politique et métaphysique.

\section{INDEX}

Mots-clés : générique, geste, membrane, normalisation, signe, virtuel

\section{AUTEUR}

\section{JOACHIM DANIEL DUPUIS}

professeur en classes prépas à l'Icam, à l'Isen et au CPMP de Lille, joachimdupuis@orange.fr 